\title{
Study on Nursing Effects of Liposuction and Volume Reduction Surgery for Lymphedema Perioperative Nursing Care
}

\author{
Wei Zheng1*, Fei Li² \\ 'Xi'an Employees' University, Xi'an 710065, China \\ ${ }^{2}$ Affiliated Hospital of Shanxi University of Chinese Medicine, Xianyang 712000, China \\ *Corresponding author: Wei Zheng, 13595024@qq.com
}

Copyright: $\odot 2022$ Author(s). This is an open-access article distributed under the terms of the Creative Commons Attribution License (CC BY 4.0), permitting distribution and reproduction in any medium, provided the original work is cited.

\begin{abstract}
Objective: To study the nursing effect of liposuction and volume reduction in the treatment of lymphedema during the perioperative period. Method: A total of 68 patients treated with liposuction volume reduction surgery for pseudolymphedema of lower limbs admitted from May 2019 to May 2020 in a tertiary hospital in Xi'an, Shaanxi Province were selected, and they were divided into observation group and control group by random grouping method. There were 34 cases in each group. The control group took routine care during the perioperative period, while the observation group performed full care during the perioperative period. The complication rate and pain degree of the two groups were compared. Results: The complication rate of the study group was $5.88 \%$, while that of the control group was $26.67 \%$. Compared with the study group, the complication rate was significantly higher than that of the control group, and the gap was statistically significant $(\mathrm{P}>0.05)$. The pain scores of patients in the study group were significantly lower than those in the control group at 3 and 6 days after surgery, and the gap was statistically significant $(\mathrm{P}>0.05)$. Conclusion: Lymphedema should be taken care of during the perioperative period of liposuction and volume reduction, which can effectively reduce the occurrence of pain and complications. It is worthy of extensive clinical promotion.
\end{abstract}

Keywords: Liposuction volume reduction surgery; Lymphedema; Perioperative period; Complications; Pain

Online publication: January 19, 2022

\section{Introduction}

Lymphedema is mainly due to the accumulation of lymph fluid in the subcutaneous tissues, which leads to lymphatic drainage obstacles and local edema ${ }^{[1]}$. Lymphedema is divided into primary and secondary. Because lymphedema is a chronic disease, when edema occurs, fatty tissue fibrosis, fatty hardening, and skin roughness occur. The clinical technology of this disease is constantly updated, liposuction volume reduction surgery is one of them ${ }^{[2]}$. This technology is widely used in various cancer types, such as lung cancer, gastric cancer, breast cancer, etc. Surgery is certainly an important treatment method, but it is also indispensable to keep the patient's vital signs stable during the perioperative period. This time, liposuction is adopted for lymphedema. The perioperative care of volume reduction surgery is discussed in detail below.

\section{Materials and methods}

\subsection{General information}

A selection of 68 patients treated with liposuction volume reduction surgery for pseudo-lymphedema of the 
lower limbs admitted to a third-class hospital in Xi'an from May 2019 to May 2020, and randomly divided them into observation group and control group, with 34 cases in each group, the male to female ratio in the control group is $19: 15$, and the age range is $32-51$ years old, with an average of $(41.26 \pm 2.13)$ years old. The male to female ratio in the observation group was 20:14, and the age range was 32-50 years old, with an average of $(41.67 \pm 2.09)$ years old. The patients and their families were aware of the purpose and precautions of this survey. The general data of the two groups of patients were not statistically significant $(\mathrm{P}<0.05)$ and were comparable ${ }^{[3]}$.

Exclusion criteria: abnormal cognitive function; organic diseases such as heart, liver and kidney; suffering from primary immune system diseases; taking glucocorticoids within the past 3 months.

\subsection{Methods}

\subsubsection{Control group}

The control group performs routine nursing care, such as preoperative health education, distributing drugs or injection therapy according to doctor's instructions.

\subsubsection{Observation group}

The observation group implements the whole process of nursing, such as

(1) Cognitive and psychological assessments of the patients are carried out before the operation, and the nursing staff patiently communicates with the patients actively. In the communication process, analyze the psychological state of the patient. For patients with anxiety and depression, stand beside the patient, and organize relevant experts to give health lectures, so that patients can transform their thinking into action in the lecture class.

(2) Thrombosis prevention: Inform patients not to over-exercise or to prevent thrombosis, wear tight elastic stockings of the wrong size to further aggravate blood circulation.

(3) Pain care: To relieve pain, start with the NRS vernier scale. When the pain reaches severe levels, opioid analgesia can be given. For other degrees of pain, use the method of diverting attention ${ }^{[4]}$.

(4) Skin care: Always maintain pressure when wrapping the bandage to avoid excessive pressure affecting blood circulation and even skin necrosis.

(5) Drainage tube care: Nursing staff check the condition of the incision twice a day, observe the nature of the drainage fluid, prevent the drainage tube from clogging, and replace the drainage bag in time ${ }^{[5]}$.

\subsection{Observation indicators}

The complications and pain levels of the two groups were compared. The degree of pain was measured by NRS vernier, 0 means no pain, 1-3 points means toxic pain, $\geqslant 4$ points mean severe pain.

\subsection{Statistical processing}

Use SPSS26.0 software to perform statistics. Measurement data are expressed as mean \pm standard deviation $(\mathrm{x} \pm \mathrm{s})$, using $\mathrm{t}$ test, counting data are expressed as frequency, using chi-square test, and $\mathrm{P}<0.05$ indicates that the difference is statistically significant.

\section{Results}

\subsection{Comparing the occurrence of complications between the two groups of patients}

Compared with the control group, the incidence of complications in the study group was significantly lower than that in the control group, and the gap was statistically significant $(\mathrm{P}<0.05)$. See Table 1 for details. 
Table 1. Comparison of the occurrence of complications between the two groups of patients [n (\%)]

\begin{tabular}{cccccc}
\hline Group & Number of cases & $\begin{array}{c}\text { Delayed incision } \\
\text { healing }\end{array}$ & Infect & Stiff joints & Total incidence \\
\hline Observation group & 34 & $1(2.94)$ & $1(2.94)$ & $0(0.00)$ & $2(5.88)$ \\
Control group & 34 & $3(8.82)$ & $5(14.70)$ & $1(2.94)$ & $9(26.47)$ \\
$\mathbf{X}^{2}$ & - & - & - & - & 5.314 \\
$\mathbf{P}$ & - & - & - & - & 0.021 \\
\hline
\end{tabular}

\subsection{Compare the pain degree of the two groups of patients}

Compared with the control group, the patients in the study group had no statistically significant pain on the first day after the operation ( $\mathrm{P}>0.05$ ), but the pain on the 3 and 5 days after the operation was significantly lower than that in the control group, and the gap was statistically significant $(\mathrm{P}<0.05)$, see Table 2 for details.

Table 2. Comparison of the pain degree of the two groups of patients $(\mathrm{x} \pm \mathrm{s})$ (points)

\begin{tabular}{ccccc}
\hline Group & Number of cases & 1d after operation & 3d after operation & 5d after operation \\
\hline Observation group & 34 & $8.69 \pm 1.09$ & $5.94 \pm 0.85$ & $3.26 \pm 0.34$ \\
Control group & 34 & $8.71 \pm 2.01$ & $7.28 \pm 0.95$ & $5.26 \pm 0.29$ \\
$\mathbf{X}^{\mathbf{2}}$ & - & 0.051 & 6.129 & 26.096 \\
$\mathbf{P}$ & - & 0.960 & 0.000 & 0.000 \\
\hline
\end{tabular}

\section{Discussion}

Local edema and paresthesia are the most common symptoms of lymphedema, which refers to a blockage of lymphatic drainage in a specific part of the human body, resulting in a continuous accumulation of lymphatic fluid in the subcutaneous tissue, causing subcutaneous fibrous connective tissue and fatty sclerosis. According to global lymphedema patient data, around 170 million lymphedema patients have liposuction volume reduction surgery, the primary goal of which is to suction away accumulated fatty tissue using a vacuum aspirator ${ }^{[6]}$. Although the condition may be treated very away, follow-up care is critical. The integration of the entire course of nursing measures can promote the patient's disease recovery on the basis of assuring surgical therapy.

The results of this survey showed that the incidence of complications in the study group was significantly lower than that in the control group, and the gap was statistically significant $(\mathrm{P}>0.05)$; The pain scores of patients in the study group were significantly lower than those in the control group at 3 and 6 days after surgery, and the gap was statistically significant $(\mathrm{P}>0.05)$. This shows that the whole course of nursing measures relieves the patient's psychological emotions before the operation, and then the patient should be informed of the precautions for surgical cooperation, and for the complications that may occur during the operation, such as thrombosis, pain, etc., the clinical routine care is only carried out in accordance with the doctor's instructions. Nursing measures are just based on the corresponding nursing measures completed by the doctor who prescribes the patient's condition. There are few preventive and interventional measures, and the service of the whole course of care is carried out with a combination of prevention and intervention dual care measures, to maximize the patient's treatment effect and control the incidence of complications.

To sum up, during the perioperative period of liposuction and volume reduction treatment for lymphedema, comprehensive care can effectively reduce complications and relieve the pain of patients. It 
is worthy of extensive clinical promotion.

\section{Disclosure statement}

The author declares no conflict of interest.

\section{References}

[1] Xin J, Sun Y, Xia S, et al., 2019, Lymphatic Liposuction Volume Reduction Surgery in the Treatment and Analysis of Primary Lymphedema of Lower Limbs. Chinese Journal of Plastic Surgery, 35(2): 6.

[2] Xin J, Sun Y, Xia S, et al., 2019, Treatment and Analysis of Lymphatic Liposuction Volume Reduction Surgery in Primary Lymphedema of Lower Limbs. Chinese Journal of Plastic Surgery, 35(2): 142 147.

[3] Dai Q, He C, Huang X, et al., 2019, Perioperative Nursing Care of Vascularized Supraclavicular Fossa Lymph Node Flap Transplantation for Treatment of Lower Limb Lymphedema. Chinese Journal of Microsurgery, 42(2): 3.

[4] Bart, Zhang W, Niu R, et al., 2019, Research Overview of Traditional Chinese Medicine Treatment of Lower Limb Lymphedema after Radical Resection of Cervical Cancer. Journal of Shanxi College of Traditional Chinese Medicine, 20(3): 4.

[5] Zhang J, Chen Z, Jiang W, 2020, The Effect of Pressure Gradient Stockings Combined with Intermittent Pressure Inflation Device to Prevent Lower Limb Lymphedema after Gynecological Tumor Lymphadenectomy. General Nursing, 18(14): 4.

[6] Ji L, Li W, Qi Y, et al., 2020, The Effect of Manual Lymphatic Drainage Combined with Air Pressure Comprehensive Therapy on Patients with Lower Limb Lymphedema. Electronic Journal of Clinical Medicine, 7(29): 1.

Bio-Byword Scientific Publishing remains neutral with regard to jurisdictional claims in published maps and institutional affiliations. 